Recepción: 11/05/2015

Evaluación: 02/06/2015

Aprobación: 10/08/2015

Artículo de Revisión

\title{
LA EDUCACIÓN COMO ACONTECIMIENTO ÉTICO: UNA ALTERNATIVA PARA EL RECONOCIMIENTO DE LA DIFERENCIA
}

\author{
Edwin Giovanni Ordóñez \\ Universidad de Nariño \\ Grupo de Investigación: HISED \\ Línea de investigación: Historia de la Educación en Colombia \\ edwingior@hotmail.com
}

\begin{abstract}
RESUMEN
El presente artículo de revisión recrea la ética como condición de posibilidad de la relación educativa como respuesta a las percepciones pedagógicas y sociales que posee el docente acerca de la diferencia en el devenir educativo. La metodología se determina en el producto de la tesis titulada: "Las representaciones sociales de los docentes acerca de la diferencia en el aula" fundamentada en el paradigma cualitativo con una perspectiva de análisis crítico y hermenéutico en el sentido de comprender cómo los docentes en sus prácticas o discursos representa categóricamente al estudiante en el aula de clases. Además, este artículo busca que los docentes y las instituciones educativas valoren la diferencia no desde la homogenización, sino desde un acontecimiento ético mediante tres aperturas: la apropiación de las condiciones sociales y culturales del medio educativo; la hospitalidad; y el reconocimiento de las singularidades y su diversidad.
\end{abstract}

Palabras Claves: Diferencia, Ética, Moral, Representación social. 


\title{
EDUCATION AS AN ETHICAL EVENT: AN ALTERNATIVE FOR THE RECOGNITION OF DIFFERENCES
}

\begin{abstract}
This article reflects on the recreation of Ethics as a possible condition of educational relationships in response to pedagogical and social perceptions that the teacher holds in terms of differences in the educational process. The methodology is determined by the product of a thesis entitled "The Social Representation of Teachers Regarding Differences in the Classroom," which is based on a qualitative paradigm with a perspective of hermeneutic and critical analysis in the sense of understanding how teachers, in their practices or speech, are categorically represented to the student in the classroom. In addition, this article seeks how teachers and educational institutions value differences, not from homogenization, but as an ethical event mediated through three courses: the appropriation of the social and cultural conditions of the educational environment; hospitality; and the recognition of their singularities and their diversity.
\end{abstract}

Key Words: difference, ethics, morality, social representation

\section{A EDUCAÇ̃̃O COMO ACONTECIMENTO ÉTICO: UMA ALTERNATIVA PARA O RECONHECIMENTO DA DIFERENÇA}

\begin{abstract}
RESUMO
Este artigo recria ética como condição de possibilidade da relação educativa em resposta às percepções pedagógicas e sociais detidas pelo docente sobre a diferença no futuro educacional. A metodologia é determinado pelo produto da tese intitulada as "Representações sociais dos professores sobre a diferença na sala de aula", baseado no paradigma qualitativo, com uma perspectiva de análise crítica e hermenêutica, no sentido de compreender como os professores em suas práticas ou discursos representa categoricamente o aluno na sala de aula. Além disso, este artigo procura que os professores e as instituições educacionais deem valor a diferença não desde o valor da homogeneização, mas, de um acontecimento ético através de três aberturas: a apropriação das condições sociais e culturais do ambiente educativo; hospitalidade; e o reconhecimento da singularidade e sua diversidade.
\end{abstract}

Palavras-chave: Diferença, Ética, Moral, Representação social 


\section{INTRODUCCIÓN}

La educación como acontecimiento ético y vital, es una relación de posibilidad e innovación para tener en cuenta la singularidad de cada uno de los estudiantes desde su cotidianidad y su propia interrelación con el medio social y cultural. Esta propuesta conduce a la valoración y el reconocimiento de cualquier sujeto y su colectivo, en tiempos y espacios específicos.

La necesidad de desarrollar el presente artículo se justificó del producto de la tesis "Las representaciones sociales de los docentes acerca de la diferencia en el aula" 1 en el cual se indagan que las representaciones sociales del cuerpo docente sobre sus estudiantes provienen moralmente del entorno cultural, económico y social, y por ende, de las normas institucionales y de las estrictas políticas homogeneizantes del Estado a través del Ministerio de Educación Nacional (MEN), por el cual, el mismo docente al igual que la institución educativa acatan las normas para su propósito laboral y pedagógico.

La diferencia es fijada y apuntada hacia un sujeto, un estudiante, un ser o un no ser, un "otro", un recibido o un ignorado, por el cual es denominada y representada en el momento de su presencia en cualquier espacialidad: el hogar, la escuela y el aula de clases, primero para ser reconocido y luego para ser abandonado a su suerte. Como consecuencia, la relación con el ser de la diferencia puede ser de dominio-imposición, de control, de persecución, de incomodidad; o por el contrario, de tolerancia, aceptación y respeto hacia el otro. Por otra parte, el representador no mira a los ojos de los otros, sino que dejan un pensar homogeneizante sin el otro, o incluso, contra el otro.

Pero la diferencia, no solo implica un conflicto social, la diferencia es lo extraño dentro de un sistema homogéneo, así que regresando a una de las preguntas transversales de esta investigación: ¿qué es la diferencia? Es preciso manifestar que, la diferencia es una condición de carácter representativo, que genera un aprieto dentro de un entorno o sistema homogeneizado; en este sentido, es posible decir que: diferente es el estudiante nuevo, por su representación como nuevo dentro del aula; de la misma manera se podría asimilar por diferente al alumno de mal comportamiento, o el alumno de origen étnico; en otras palabras, son diferentes aquellas personas que no generan representaciones homogeneizadas preestablecidas socialmente.

Además, en un contexto real, el docente omite reconocer al estudiante y su colectivo, puesto que representa la diferencia en sus cuatro componentes: el

1. Carolina Carvajal, Las representaciones sociales de los docentes acerca de la diferencia en el aula. Tesis de Maestría en Educación en Universidad de Nariño (2015). 
juicio moral (normas, valores, leyes), el contexto sociocultural (aula, familia, localidad, barrio, ciudad, región), el ego modelo (métodos, esquemas de evaluación) y los conceptos valorativos (logros, comportamientos). Como consecuencia de ello, la singularidad del estudiante se ha visto opacada, invisible y ausente de miradas por parte del docente, porque no se tienen en cuenta las condiciones personales, familiares y sociales con las que viven $y$ conviven por los efectos de sus representaciones sociales.

Como trasfondo se complementaran discursos textuales de grandes pensadores como Bárcena y Mélich (2014), Lévinas (1977), Skliar (2005, 2007, 2011,2013), entre otros, que facilitan la comprensión y la concepción de la diferencia bajo una argumentación de otro tipo de lenguaje antropológico, filosófico y educativo acorde a la realidad.

Finalmente, se recrea la correspondiente interrelación de supuestos, conceptos y preceptos teóricos que ameritan la dinámica propia de su accionar en las instituciones educativas mediante tres aperturas fundamentales para el acontecer ético y vital como son: la apropiación de las condiciones sociales y culturales del medio educativo, la hospitalidad, el reconocimiento de las singularidades y su diversidad, ya que son fundamentales para una educación desde y para la vida.

\section{EL UNIVERSO EDUCATIVO MORAL}

Figura 1. Pirámide representativa relación docente-estudiante.

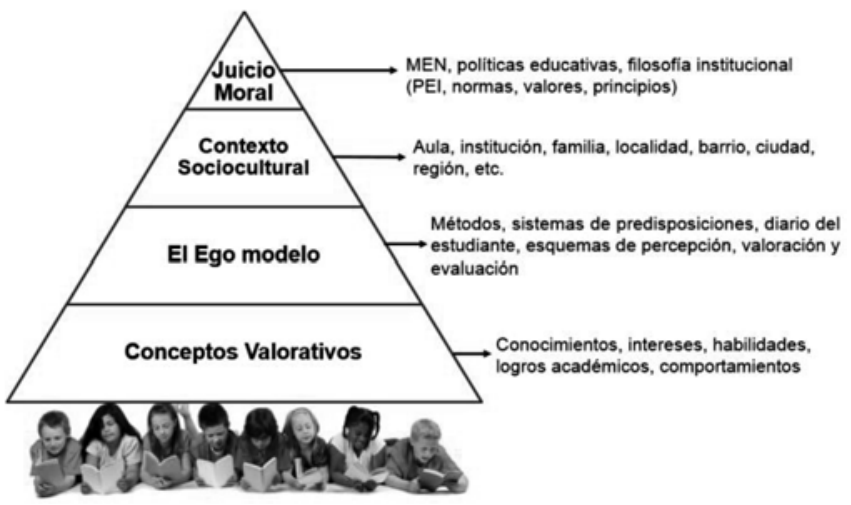

Fuente: Carvajal, p. 84.

En la figura 1, la cúspide es el «juicio moral» ejercido por el docente y la institución educativa bajo la presión e influencia de la poderosa moral, que desde un sentido etimológico son las reglas, posicionamientos, normas o consensos por las que se rige, regula y juzga el comportamiento o la conducta 
de un ser humano en una sociedad. Para Mélich, la moral es "un marco sígnico-normativo propio del espacio público y de una cultura concreta en un determinado tiempo de la historia" 2 , y en otro sentido, la moral como "una gramática compuesta de signos, categorías, principios, ritos, hábitos y valores (...) que fabrica, ordena y organiza el mundo"3. Y es ésta gramática la que nos permite acercar y representar al ser humano.

Las entidades sociales como el estado, la escuela, la familia, la iglesia, los medios de comunicación, las costumbres culturales y "la mismidad" 4 . Son aquellas que han atribuido el universo moral para inventar y banalizar las concepciones sobre la vida cultural, educativa y social, de las comunidades, las minorías y de los diversos tipos de población. Al igual esta moralidad presenta orificios, vacíos y limitaciones en la manera de definir al ser y su alteridad en base a su realidad.

El estado a través de la norma y la ley, aunque cargada de fetichismo, corrupción, omisión y otros males políticos, posee una colonización instrumental y sistemática de todos y cada uno de los aspectos de la existencia humana, que son implementados mediante dispositivos de control llamadas en adelante "representaciones legales", como aquellas formas legítimas que constituyen, categorizan y representan a los seres humanos, como una forma de imposición de saber y verdad en cuanto a su tipo de ser, sus características físicas, psicológicas y emocionales; y su diversidad como lo multicultural y lo pluriétnico, entre otras. Además, estas representaciones legales se instituyen en ordenanzas y proposiciones que especifican y determinan aquello que es aceptado al interior de la sociedad o aquello que debe ser controlado para tratarlo y normalizarlo mediante prácticas homogeneizadoras.

A nivel educativo, el MEN en representación del estado colombiano y los establecimientos educativos, entre otros, bajo efectos de poder y obediencia despliega normas, principios, valores, códigos, deberes, objetivos, propósitos o fuentes de representación homogeneizantes hacia las dinámicas educativas y los actores sociales (estudiantes, docentes y comunidad educativa en general), en la cual generan formas de denotar y representar mediante discursos, dispositivos y mecanismos legales, haciendo caso omiso a sus particularidades individuales.

Es por ello, que la moral influye a la mismidad docente para mostrar fidelidad en cuanto a su estilo pedagógico y a su metodología que puede ser

2. Joan-Carles Mélich, La Lógica de la Crueldad (Barcelona: Herder, 2014), 135.

3. Mélich, 55.

4 Aquella condición que le permite a una persona o entidad "ser uno mismo" con una identidad en función del encuentro y la existencia de la alteridad del Otro. 
asignada por el sistema educativo, la institución o desde su particularidad; ésta llega a ser una forma inicial de control y representación hacia los estudiantes.

Como segundo componente se encuentra el «contexto sociocultural» (ver figura 1)en el cual vive y convive el estudiante, dentro y fuera de la institución educativa como la vivienda familiar, la localidad, el barrio, la ciudad, la región, etc.

Cualquier institución educativa en base de su filosofía y el proyecto educativo institucional busca el logro de unos fines y objetivos en común dentro del sistema escolar. Para ello, se elabora el proyecto educativo institucional (PEI), que pretende que los estudiantes se conviertan en buenos ciudadanos, críticos, y competentes pero con comportamientos homogéneos, quienes a su vez, faciliten la construcción de sociedades silenciosas dentro de los marcos del orden institucional. Un ejemplo de ella consiste en aquella norma que impone a los individuos de una escuela como son: un uniforme, un lenguaje, un horario, un uso controlado del tiempo, una evaluación, entre otras cosas.

Reconocer el contexto sociocultural desde la institución educativa y su entorno implica un alto grado de tolerancia en caso de una existencia de alguna cultura representada en la diferencia dentro del sistema social. Por lo tanto, los docentes tienden a no aceptar la posibilidad de conocer esa cultura diferente, es decir, conoce o asimila su existencia, pero no la convive y, en esa perspectiva, no la asimila dentro de su espacialidad. Además, la diferencia siempre se queda en la mismidad como si fuera su sombra, no por voluntad propia, sino por la fuerza y el carácter de la norma.

Para tal caso, el aula de clases es el primer escenario donde el docente genera y percibe las representaciones sociales, denominadas como "conjuntos de conceptos, enunciados y explicaciones originados en la vida diaria, en el curso de las comunicaciones interindividuales"5, para visibilizar a algunos y ocultar a otros, en la manera de cómo los sujetos son presentados ante el mundo social, y apuntados en la diferencia o en su caso, el diferencialismo ${ }^{6}$ hacia cada estudiante en cuanto a sus capacidades académicas y actitudinales cotidianas, resultado de su contexto sociocultural.

Algunas de las representaciones sociales con respecto a la diferencia se observan en los siguientes discursos de los maestros:

5. Moscovici, Serge. El psicoanálisis, su imagen y su público (Buenos Aires: Huemul, 1979), 182.

6. El diferencialismo comprendido como una diferencia no aceptada y no reconocida, en la forma de denominar la diferencia partir de una connotación peyorativa y subalterna. Por ejemplo: "la mujer sea considerada el problema en la diferencia de género, que el negro sea considerado el problema en la diferencia racial, que el niño o el anciano sean considerados en el problema de la diferencia de edad..." (Carlos Skliar. La educación que es del otro (Medellín: Artes \& Letras Ltda., 2007), 98. 
Lógico es que todos son diferentes, todos, porque cada persona es un mundo aparte los veo como diferentes porque uno se encierra en treinta mundos diferentes, por ejemplo tengo excelente estudiantes y hay veces que ellos decaen por los problemas internos de su casa

(...)

yo diría que cada personita de estas es un mundo diferente, cada quien tiene sus actitudes y aptitudes donde por ejemplo encontramos estudiantes que tienen muchas capacidades, en los deportes, se caracterizan por tener un gran potencial para desarrollarse en las disciplinas del microfútbol, futbol, baloncesto algunos son patinadores, son nadadores, trabajan con tenis de mesa y algunos otros por ejemplo como se les puede llamar comúnmente son los buenos estudiantes o los nerdos y en ellos también encontramos este tipo de situaciones ${ }^{7}$.

No obstante, esas representaciones sociales también tienen una percepción sobre su condición externa y su futuro:

No tienen un proyecto de vida, se conforman con lo básico, para ellos es importante un tres y punto, no se esfuerzan por sacar notas más altas, es raro el estudiante que diga yo quiero ser el mejor del curso. Lo atribuyo al medio, básicamente al nivel de los padres (...) la descomposición familiar es grande, tenemos hasta padres que son recicladores ${ }^{\mathbf{8}}$.

Por tal manera, el salón de clases no es un espacio neutral, puesto que depende de las representaciones sociales percibidas por los docentes, es decir, esta espacialidad no escapa a las comparaciones que provienen no sólo de ellos, sino del entorno económico, social y cultural como procedencia, barrio, entorno familiar (padres casados, descomposición, madre soltera, acudiente, hogar sustituto), condiciones económicas (pobreza), etnia, población vulnerable (desplazados, víctimas de violencia), entre otros factores. A partir de este punto, también se deriva el espacio en el cual el docente conjetura y obtiene un sinnúmero de representaciones sociales relacionadas con sus estudiantes.

El tercer componente, conocido como «el ego modelo» (ver figura 1), se refiere a la autonomía del docente para controlar, domesticar o vigilar las acciones de sus estudiantes, mediante técnicas, métodos, esquemas de evaluación y promoción- Estos elementos son términos que sugieren "el deseo de ser fiel a una forma sistemática evaluable y mensurable de las intervenciones educativas"9, por lo que, el docente necesita que sus estudiantes respondan

7. Carvajal, 42-43.

8. Carvajal, 103.

9. Fernando Bárcena y Joan-Carles Mélich. La educación como acontecimiento ético: natalidad, narración y hospitalidad (Buenos Aires: Miño y Dávila, 2014), 83. 
a su desempeño como "todos iguales, todos buenos, todos juiciosos" en las actividades académicas cotidianas. También como un alumno ideal: hecho a imagen y semejanza del docente; y "reflejo de si", como lo expresa abiertamente una docente:

El estudiante es dedicado, maneja la diferencia, él es como el reflejo de lo que yo soy, me gusta con una película, no sé si la han visto, Manos Milagrosas, hablaba que todos podemos hacer lo mismo, pero uno lo puede hacer mejor, entonces manejo eso usted haga las tareas, pero trate de hacerlas mejor, por eso es la competencia, entonces él es el reflejo de lo que yo soy, excelente en las tareas...10

Existe la creencia o la rutina, que para entender mejor las explicaciones en el salón de clases, el lenguaje del docente debe ser homogéneo en el sentido del uso del lenguaje para cualquier contenido en su respectiva área. De este modo, se supone que las ideas o conocimientos que puede imponer el docente serán asimilados con mayor facilidad por parte de los estudiantes. Sin embargo, este proceso no es tan simple ya que:

implica mayor complejidad porque: a). estudiantes y docentes deben compartir el mismo código lingüístico (monolingüismo) y un código cultural común en el aula; b). todas y todos los estudiantes deben tener las mismas capacidades cognitivas; $\mathrm{y}, \mathrm{c}$ ). compartir los mismos códigos disciplinarios ${ }^{11}$.

El cuarto y último componente es el «concepto valorativo» (ver figura 1), por medio del cual el docente representa al estudiante mediante los niveles de aprendizaje, los logros, los conocimientos, la responsabilidad, la participación, la colaboración con el docente, los buenos modales, el cumplimiento de tareas, entre otros aspectos de valoración académica.

Un estudiante es considerado como un número de lista, un apellido, un nombre y una foto dentro de un cuaderno de notas, un diagnóstico u observación, las cuales son dispositivos de clasificación del docente para observar las capacidades, los logros académicos y las competencias de cada estudiante, y luego, permite ubicarlo en diferentes categorías y eufemismos como: buenos, malos, juiciosos, desobedientes, sumisos, humildes, callados, líderes, activos, pasivos, responsables, irresponsables, ordenados, desordenados, etc.

El indicador más común y utilizado para medir este concepto valorativo es la "nota", aspecto que condiciona pedagógicamente al estudiante de acuerdo a unos criterios unificadores establecidos no solo por el docente, o la institución sino por los lineamientos del MEN.

10. Carvajal, 57.

11. Carvajal, 80. 
Para el docente, educar en la diferencia genera angustias, soledad, impaciencia, esfuerzos y estados de exaltación, quien pretendiendo resolver tales tensiones, se encuentra impotente frente a la resolución de las mismas. Y aunque lo diferente implique una amenaza al sistema preestablecido, el "otro", "el diferente", en buen número, termina sometiéndose a sus requerimientos. No obstante, en ese proceso algunos "otros" generan resistencia que sería la negación de sí o la defensa de sí, que se caracteriza por generar dinámicas de tensión (de la relación docente-estudiante) que se manifiestan en diferentes grados de violencia y que se desarrollan en el aula de clase.

Sin embargo, este componente tiene un sentido oculto, puesto que se encuentran las acciones más destacadas de los educandos como: las destrezas, las capacidades, los conocimientos, los intereses y las necesidades específicas; las formas de interacción, entre otras. Cada estudiante se caracteriza como un ser único e irrepetible en cuanto a su singularidad (o su alteridad). Sin embargo, dichas acciones personales se ha vuelto opacas, invisibles y ausentes de mirada porque no se tienen en cuenta las condiciones personales, familiares y sociales con las que conviven por los efectos de las representaciones de los docentes.

Adicionalmente, el sistema educativo colombiano se ha transformado en un modelo de fabricación de seres humanos al servicio del modelo imperante o el sistema capitalista mundo que ha orillado a los estudiantes hacia una búsqueda de modelos de individualismo, competitividad y/o originalidad. Dichos aspectos condicionan la utilidad de la gente desde dos ámbitos: por un lado para posicionarse en las categorías de mejores con privilegios que los demás esperan y por otro lado en un desinterés en la vida escolar y una crisis de fe en el futuro profesional. Por lo que Bárcena y Mélich plantean que si "la educación es una fabricación, entonces la identidad propia deja de construirse, y aparece el sujeto orgulloso, que escapa al tiempo, surge el sujeto substancializado, totalitario"12.

\section{LA EDUCACIÓN COMO ACONTECIMIENTO ÉTICO Y VITAL}

La educación como acontecimiento ético y vital, es una propuesta alternativa que permite cruzar la frontera para situarse en la realidad del "Otro", es decir, "antes y después del encuentro cara y cara con el otro, un encuentro absolutamente singular y único"13 en tiempos y espacios específicos. Para alcanzar este propósito, éste debe efectuarse lejos de toda brecha y hostilidad moral

12. Bárcena y Mélich, La educación como acontecimiento ético, 82.

13. Bárcena y Mélich, 13. 
que solo busca dominio, persecución y control hacia los sujetos, y además, no mira a los ojos de los otros, sino que dejan un pensar homogeneizante sin el otro, o incluso, contra el otro.

La ética no debe entenderse como algo que está en el fundamento epistemológico u ontológico de las ciencias humanas, sino por lo planteado por Zenón de Citio que definió el ethos como "una fuente de la vida, de la que manan los actos singulares". En esta fuente de vida se encuentra los caracteres propios como: el hábito, el temperamento, la conciencia, y los modos de ser, lo que conlleva que para aplicarse debe realizarse mediante acciones como: juzgar el comportamiento o la conducta de los individuos y la plena moral de manera relativa.

La ética y la moral, son palabras con significados divergentes; la moral es aquella que posee leyes homogeneizantes propias de la mismidad, la globalidad y la totalidad; por otra parte, la ética es la columna vertebral de nuestras acciones, nuestras vidas y nuestras conciencias, como sostiene Savater "el arte de vivir, el saber vivir"14, que nos tumba y nos transforma como agentes constructores de humanidad junto a otros seres humanos frente a los marcos legales y morales previamente instituidos.

Los acontecimientos morales y éticos surgen en la relativa tensión entre el mundo y la vida, la ley y la ruptura, la obediencia y la resistencia, la utopía y la realidad. Bárcena y Mélich establecen que "heredamos un mundo, pero configuramos, creamos nuestra vida; la configuramos desde el mundo, pero, a veces es necesario, configurarla contra el mundo. No es posible una vida sin mundo, pero una vida reducida al mundo es una vida invisible"15.

Para ejecutar la educación como acontecimiento ético se propone tres aperturas vitales como: la apropiación de las condiciones sociales y culturales del medio educativo, la hospitalidad, y el reconocimiento de las singularidades y su diversidad.

\subsection{La apropiación de las condiciones sociales, culturales del medio institucional}

Toda institución determina claramente su misión, sus objetivos y principios en base al proyecto educativo institucional (PEI) y para adecuarla en el acontecimiento ético, no se trataría de buscar reformas educativas, políticas de innovación que permitan ubicar los programas educativos acordes a las

14. Fernando Savater, Ética para Amador (Barcelona: Ariel, 1991), 9.

15. Bárcena y Mélich, 266. 
necesidades e intereses locales y regionales, en una nueva representación y consideración. Sino más bien, prestar atención a las rutas o estrategias de desarrollo para ver si concuerdan cabalmente con lo que enseña en el espacio escolar y lo que reclama el sujeto educativo y la comunidad local, al igual que "comprendan y reconozcan la heterogeneidad como característica de las aulas de clase; empezando con la diferencia socioeconómica, racial, religiosa, cultural entre otras"16. Dicho proceso requiere una responsabilidad a medida de las condiciones que caracteriza a un sujeto educativo, y "más proclive a la escucha del otro que a la visión o contemplación neutral y objetiva de lo inteligible del mundo"17.

Por lo tanto, el aula de clases es el contexto más próximo para que el docente pueda identificar la realidad escolar seguido el ambiente institucional y la comunidad en particular. Por lo que Latorre y Suarez sugieren:

"Es precisamente en el aula de clases donde hay que cambiar las relaciones de poder y la estructura centralizada en el docente y en la institución que generan conflictos en el campo interpersonal e intrapersonal de cada estudiante (...) es en el aula donde la misión de cada proyecto educativo se hace realidad como una formación apoyada en componentes pedagógicos coherentes con una concepción crítica y personalizante de la educación" ${ }^{\prime 18}$.

La interculturalidad es también un aspecto a tener en cuenta en la apropiación del contexto institucional acorde, ya que procura al "otro" una presencia o realidad a ocupar, sin que este pierda sus pautas o representaciones sociales que lo hacen diferente, sin la necesidad de ser colonizado o excluido. Por lo tanto, la identidad genera que la diferencia no tenga ningún tipo de jerarquización dentro del sistema, sino que se concibe dentro de las mismidades, como el espacio de reconocimiento y aceptación de la diferencia.

\subsection{La hospitalidad}

La hospitalidad es la responsabilidad ética de la acogida, la bienvenida, el recibimiento, el reconocimiento, el ethos "la morada" y la presencia real del y hacia el "Otro", y para acogerlo se materializa al conducirlo como un huésped, alguien a quien hay que introducir y acompañar, sin importar el lugar donde se desarrolle su vida, su existencia, su lenguaje, sus valores propios; y al mismo tiempo pueda compartir con su colectivo, sus propios pensamientos, imágenes

16. Armando Zambrano, La mirada del sujeto educable: la pedagogía y la cuestión del otro (Cali: Nueva Biblioteca Pedagógica, 2001), 74.

17. Emmanuel Lévinas, Totalidad e Infinito. (Salamanca: Sígueme, 1977), 262.

18. Helena Latorre y Pedro Suárez, La evaluación escolar como mediación: Enfoque sociocrítico (Bogotá: Fundación Francisca Radke, 2001), 220. 
y voces en diferentes ambientes de acogida como la familia, las amistades, la comunidad y la escuela ya que "no hay leyes en la ley de la hospitalidad porque en ella se declara la abertura, el recibimiento, la acogida al otro (...) porque es el Otro el que decide si vendrá o no vendrá"19.

Por lo anterior, un ambiente de hospitalidad en conjunto de la educación como acontecimiento ético y vital consistiría en recrear una educación imprescindible y de formación humana e integral, en que "cualquiera" y "cada uno" de los miembros -la gente- que forma parte de la comunidad educativa estén cargados de energías, impulsos y empujes ante sus labores cotidianas y su interacción con sus pares educativos. Asimismo, hablar de energías consiste en un sinnúmero de acciones sencillas como: dar la bienvenida, saludar, acompañar, compartir, conversar, dar testimonio, permitir, posibilitar, dejar, dar, observar, leer, jugar, atender, o escuchar.

\subsection{El reconocimiento de las singularidades y su diversidad}

Cada una, cada uno es quien es, que no hay dos iguales, que cada quien tiene que ser visto, entendido, y reconocido según quién es. Que cada quien tiene su propia historia, su propio devenir, su propio futuro, y que reconocer esto, y cuidarlo en educación debería ser lo primero.

José Contreras

El reconocimiento de la singularidad y su diversidad es el aspecto más importante en la educación, en el cual contemplamos el auténtico actuar humano, "como una ventana que nos abre el mundo y a las mentes de otros" 20 , y esta ventana es el lenguaje, la figura, el gesto, el pensamiento y la palabra en el seno de una esfera pública asentada en la diversidad. Dicha acción conmueve en sí mismo y para los demás el acontecimiento ético, en tiempos y contextos específicos.

Un aspecto a tener en cuenta, es la noción de diversidad, que actualmente se encuentra relacionada con las gramáticas como: integración, inclusión, multiculturalidad, tolerancia, etc. Estas gramáticas o propagandas políticas han entrado a la escena educativa para banalizar, desviar e ignorar el lenguaje, la filosofía y la pedagogía del "ser humano" mediante un "ser universal y abstracto", que únicamente tienden a establecer límites, fronteras, y encerrar desigualdades hacia el "Otro" y los "Otros", en relación a los grupos diversos sociales y culturales como lo son etnias, población LGBTI, comunidad sorda, etc.

19 Carlos Skliar, Lo dicho, lo escrito, lo ignorado: ensayos mínimos entre educación, filosofía y literatura (Buenos Aires: Miño y Dávila, 2011), 184.

20 Bárcena y Mélich, 76. 
Como ejercicio, la noción de singularidad y diversidad deben ser entendidas y comprendidas como nuevas formas de relacionarnos con todas las personas y nosotros mismos, traspasando los significados fetiches de cualquier palabra que fije diferencias o diferencialismos para crear nuevas etiquetas. Como lo sostiene Skliar "Otro es sólo una palabra, no más que una palabra, de acuerdo, pero no cualquier palabra. En realidad ninguna palabra es cualquier palabra"21. Es decir, cada palabra que representa a la diferencia es a la vez todo o nada.

Para redefinir, una singularidad es un cuerpo, un rostro, un nombre, un pensamiento, un movimiento, que los hacen únicos e irrepetibles en el mundo que lo rodea. Tanto la presencia de un singular en el ámbito educativo pone al descubierto múltiples formas de ser, ritmos de aprendizaje, habilidades, proyectos de vida tanto personal como social para el desarrollo de su autonomía, lo mismo con la interacción con sus compañeros de clase, con sus docentes, su entorno familiar y los demás miembros de ese núcleo social.

Por lo anterior, para que un docente pueda comprender la singularidad del estudiante en cuanto a sus pensamientos, sus intenciones, sus deseos, sus creencias en el marco de su proyecto personal de vida, se argumentaría en palabras de Freire "aquel docente que comienza, precisamente, escuchando y observando con toda su atención al Otro, desde la íntima convicción de que el otro vale, de que el otro es sabio y aporta al conocimiento"22. Es decir la capacidad del docente en la apertura, prudencia y escucha al otro, sobre lo que de verdad desea y quiere en el marco de un proyecto personal de vida en un espacio y tiempo concreto.

Por lo tanto, el mismo docente debe limitarse de toda fabricación, duplicación, moldeamiento de sujetos educativos iguales y ponerse en relación con todo lo que sea otro, es decir, dejar a sus estudiantes como dice Skliar "estar siendo múltiples, intraducibles e imprevisibles en el mundo (...) como humanos hechos de diferencias, y no para acabar con ellas, sino para mantenerlas, sostenerlas en su más inquietante y perturbador misterio"23.

Igualmente, percibir una representación social en el acontecer ético depende de nuestra forma de interpretar la realidad de cada uno de los sujetos educativos y su ambiente social, educativo y cultural, y también de nuestros

21. Carlos Skliar, "Educar entre unos y otros, conocidos y desconocidos", Ambits de psicopedagogía, revista catalana de psicopedagogía y educación, ISSN 1885-6365, No. 38 (2013), 20-26.

22. Paulo Freire, La educación como práctica de la libertad (Madrid: Siglo XXI, 1989), 109.

23. Carlos Skliar. "Poner en tela de juicio la normalidad, no la anormalidad. Políticas y falta de políticas en relación con las diferencias en educación”, Revista Educación y Pedagogía, Universidad de Antioquia, Facultad de Educación, Vol. XVII, N. ${ }^{\circ} 41$ (2005), 11-22. 
sentimientos que se inclinan a querer observar, en lo que es, lo que deseamos, tememos o pensamos, independientemente de toda selección moral de las particularidades de cada individuo y su colectivo, y a la vez, suspender toda tela de juicio para poder percibir su presencia e intuición, lo que manifiesta, muestra, quiere, siente, necesite, aspira y sueña cada singularidad.

Por otra parte, la diversidad es aquella noción que reconoce y valora al auténtico actuar humano desde un sendero de pluralidad de deseos, aspiraciones, propósitos, teniendo en cuenta las condiciones que los diferencia y que los iguala, a través de su forma, su figura, su quinesia, su pensamiento y en general: sus acciones individuales y colectivas.

Como mecanismo de apropiación y vinculación de la singularidad y su diversidad, la pedagogía debe conformar nuevos trayectos basados en la ética, esto es, pautas que atiendan y prioricen el valor humano, sobre cualquier otro tipo de precepto social, político y económico. Desde la consolidación de la educación como principal paradigma de integración, los individuos deben ser asumidos como seres humanos, los cuales, tienen el derecho de establecer representaciones colectivas o individuales que no alteren la convivencia y la preservación de la especie. Es así como el educador, o mejor, el docente, debe ser:

Una respuesta al llamado del "otro", a la iniciativa del otro, a una exigencia de responsabilidad de la que no me puedo desprender, anterior a toda buena voluntad o deontología profesional. El otro (educado) es una exigencia primaria que se impone desde sí, desde su autoridad, es una solicitud que me toca desde su miseria y desde su grandeza ${ }^{24}$

\section{Conclusiones}

La educación en el marco de la ética, intenta crear modelos alternativos para valorar la diferencia sin la necesidad de generar jerarquizaciones para la subordinación o sometimiento del otro; lamentablemente el juicio moral se ejerce bajo la presión de la norma y la prolongación de la homogeneización dentro de las instituciones educativas.

Igualmente, el acontecer ético evoca el comprender sobre nuestros actos, nuestras acciones y nuestra mismidad, como dice Bárcena y Mélich inspirándose en Hannah Arendt "un poder abierto a la fuerza de lo que hace"25, busca la resistencia hacia el devenir y el porvenir incesante de las costumbres y las hostilidades morales, que solo conoce leyes homogeneizantes y rutinarias, y

\footnotetext{
24. Pedro Ortega Ruiz, "Educar es responder a la pregunta del otro". Boletín Redipe, Revista Edetania, No. 824 (2013), 15-28.

25. Bárcena y Mélich, La educación como acontecimiento ético, 80.
} 
son aquellas generadoras de representaciones sociales, de marca, de dominio, control y persecución hacia los sujetos, bajo la lógica del sistema legal.

El estilo pedagógico no va, ni se presenta, en una sola dirección del docente al estudiante, sino que hay que imponer la liberación de las jerarquías dentro del aula, desconociendo la norma de la mismidad, y la marginación de la diferencia. En esta medida, el entorno educativo será considerado libre y por ende heterogéneo, en el cual, el imperio de la educación proteja las diferentes representaciones creadas por las relaciones sociales de los sujetos, dentro de sus entornos inmediatos.

La hospitalidad en el ámbito educativo es un factor primordial para concebir el proceso formativo como una responsabilidad y acción ética. La acogida de aquellos procesos de subjetivación que laten en las periferias de lo académico constituye una apertura a una educación cotidiana y vital, dispuesta a aprender del otro, de esa relación, de esa excedencia en lugar de la negación que es posible, al recibir y acoger las diferencias. Así se piensa en una educación a partir del otro como un acontecimiento ético que se gesta en común presencia; una educación presta a esa escucha y emergencia.

Para concluir, la educación como acontecimiento ético evoca la creación y la recreación de un mundo nuevo de posibilidades, una pedagogía del reconocimiento al/del otro y la diferencia como posibilidad de cada cual siga su propio proceso, el más humanizador y personal; valorar que cada uno/una manifieste, desarrolle y ponga en juego su alteridad, su singularidad y su diversidad como parte de su formación y desarrollo integral. 


\section{REFERENCIAS BIBLIOGRÁFICAS}

Bárcena, Fernando y Mélich, Joan-Carles. La educación como acontecimiento ético: natalidad, narración y hospitalidad. Buenos Aires: Miño y Dávila, 2014.

Carvajal, Carolina entre otros. Las representaciones sociales de los docentes acerca de la diferencia en el aula. Tesis de Maestría en Educación en Universidad de Nariño, 2015.

Contreras, José. "Percibir la singularidad, y también las posibilidades, en las relaciones educativas: ¿Una pedagogía de la singularidad?" Curso “Pedagogías de las diferencias”, Flacso, cohorte 2015.

Freire, Paulo. La educación como práctica de la libertad. Madrid: Siglo XXI, 1989.

Latorre, Helena y Suárez, Pedro. La evaluación escolar como mediación: enfoque sociocrítico. Bogotá: Fundación Francisca Radke, 2001.

Lévinas, Emmanuel. Totalidad e infinito. Salamanca: Sígueme, 1977.

Mélich, Joan-Carles. La lógica de la crueldad. Barcelona: Herder, 2014.

Moscovici, Serge. El psicoanálisis, su imagen y su público. Buenos Aires: Huemul, 1979.

Ortega Ruiz, Pedro. "Educar es responder a la pregunta del otro". Boletín Redipe, Revista Edetania, No. 824 (2013).

Savater, Fernando. Ética para Amador. Barcelona: Ariel, 1991.

Skliar, Carlos. "Educar entre unos y otros, conocidos y desconocidos", Ambits de psicopedagogía: Revista Catalana de Psicopedagogía y Educación, ISSN 1885-6365, No. 38 (2013).

Skliar, Carlos. Lo dicho, lo escrito, lo ignorado: ensayos mínimos entre educación, filosofía y literatura. Buenos Aires: Miño y Dávila, 2011.

Skliar, Carlos. La educación (que es) del otro. Medellín: Artes \& Letras Ltda., 2007.

Skliar, Carlos. "Poner en tela de juicio la normalidad, no la anormalidad. Políticas y falta de políticas en relación con las diferencias en educación", Revista Educación y Pedagogía, Universidad de Antioquia, Facultad de Educación, Vol. XVII, No. 41 (2005).

Zambrano, Armando. La mirada del sujeto educable: la pedagogía y la cuestión del otro. Cali: Nueva Biblioteca Pedagógica, 2001. 